\title{
0178 INTERNATIONAL TRAUMA TRAINING: TEACHING AND EVALUATING PHYSICIANS IN A CROSS CULTURAL SETTING
}

A Sisley, E Marcolini, M El Shinawi, ${ }^{*}$ M McCunn, M El-Setouhy, T Scalea, J M Hirshon Correspondence: General Surgery Department - Faculty of MedicineAin Shams University, Abbasyia, Cairo 11566, Egypt

\subsection{6/ip.2010.029215.178}

Background Trauma is a leading cause of death and disability worldwide. Training and evaluation of physicians trauma skills and knowledge is more difficult in cross cultural settings due to language and educational custom barriers.

Methods Over 18 months, 124 Egyptian physicians participated in a U.S./Egypt jointly developed 5 day training course taught in English, teaching assessment and management of trauma patients Sequential Trauma Education Programs. Participants were evaluated with four Objective Structured Clinical Evaluations (OSCE) composed of critical and noncritical elements and multiple choice examination (MC). Performance on the two testing modalities was compared. Students completed a post course survey evaluating the course.

Results Of the 124 participants, 110 (88.7\%) scored $>70 \%$ on the MC. Of 115 participants with complete OSCE, 67 (58.3\%) passed all critical OSCE components on first attempt. Overall correlation coefficient for the total scores on all four OSCEs and the complete $\mathrm{MC}$ was $\mathrm{r}=0.18$. Using a Likert scale survey respondents $(\mathrm{N}=116)$ scored the OSCE higher than the MC in terms of usefulness in helping them learn the material ( $9.1 \mathrm{vs}$ $8.3, p<0.001$ ), accurately testing skills and knowledge (8.3 vs 7.7, p<0.005). 114 respondents, 57 preferred OSCEs, 9 preferred $\mathrm{MC}$ and 48 had no preference.

Conclusions Poor correlation between OSCE and MC scores indicates that standard using a multiple choice exam may inadequately assess students in a cross cultural setting. Consideration must be given to alternate evaluation tools to optimise training. 